\title{
Molecular Characterization of HIV-1 And Drug Resistance Among HIV-1 Infected Patients Attending Kayanza District Hospital, Burundi
}

\author{
Pascal BUTOYI ${ }^{1 *}$ Eddy O. Odari ${ }^{1} \quad$ Alex Maiyo $^{2} \quad$ Rency Lel $^{2} \quad$ Raphael W. Lihana $^{2}$ \\ 1.College of Health Sciences, Department of Medical Microbiology, Jomo Kenyatta University of Agriculture \\ and Technology, P.O. Box 62000-00200 Nairobi, Kenya \\ 2.Kenya Medical Research Institute, Nairobi, Kenya
}

This study was funded by the East Africa Public health Laboratory Network Project (EAPHLNP)/Burundi.

\section{Abstract}

Virological failure in management of HIV-1 infection has been reported to be between 11 to $24 \%$ after 12 months of treatment. Out of these, acquired or transmitted drug resistance mutations have been reported at $71 \%$ to $90 \%$ in Sub-Sahara Africa. In this cross-sectional study we aimed to determine virological failure and drug resistance mutations in HIV-1 infected patients on ART attending Kayanza district hospital, Burundi. Patients were recruited using a purposive sampling technique. After informed consent, $4 \mathrm{~mL}$ of venous blood was collected from each patient. The blood was separated into plasma and cells for various laboratory assays. Plasma viral loads were quantified using the Abbott m2000rt system. Polymerase chain reaction using gene specific primers was done after extraction of nucleic acids from plasma with $>1000$ copies/ $\mathrm{mL}$, followed by sequencing of all amplified samples. Drug resistance was determined using the IAS and Stanford University database, with phylogenetic analyses done using the neighbor joining method.Two hundred patients were recruited; $13 \%$ of the respondents had virological failure associated with multiple sex partners (adjusted odds ratio (aOR, $0.154, \mathrm{p}$ $=0.016)$ and irregularity in taking medications (aOR: $0.4, \mathrm{p}=0.014)$. Fifteen samples were successfully sequenced; $80 \%(12 / 15)$ were HIV-1 subtype C, $7 \%(1 / 15)$ subtype A, and $13 \%(2 / 15)$ were HIV-1 subtype A1. Of these, $87.5 \%$ had at least one mutation (NRTI or NNRTI), while $12.5 \%$ did not carry any Drug Resistance Mutations. The most common drug resistance mutations were M183V, T215V M41L, E44D, L74I, L210W and $\mathrm{K} 65 \mathrm{R}, \mathrm{K} 103 \mathrm{~N}, \mathrm{Y} 188 \mathrm{H}$. The prevalence of virological failure was established at $13 \%$.Our findings showed possible gaps in the last $90 \%$ of the $90-90-90$ WHO target by 2020 . The results highlight the need for intense viral load and resistance testing for patients to improve overall treatment outcome. Some strategies are needed to improve adherence counselling and drug resistance mutation testing should be implemented to monitor HIV-1 patients on ART in Burundi.

Keywords: HIV-1, antiretroviral therapy, Virological failure, DRMs, Burundi.

DOI: $10.7176 / \mathrm{JBAH} / 9-10-05$

Publication date:May $31^{\text {st }} 2019$

\section{Introduction}

The increasing use of ART has increased the lifespan of HIV infected persons and thus the lifespan of such a population has now moved closer to HIV non infected populations (Nsanzimana et al., 2015). Despite of these successes, Virological failure have been reported in many studies one year after initiation of HAART in the patients on ART despite effective combination therapy. This will inevitably be accompanied by the emergence and transmission of drug resistance viruses (Hamers et al, 2013). Also, even when optimal adherence to therapy is supported ,Virological failure at some point during the ART have been reported in $10 \%$ to $30 \%$ of individuals receiving a first-line NRTI/NNRTI-containing treatment regimen and the majority of these individuals are expected to acquire NRTI- and/or NNRTI-resistant viruses in sub-Sahara Africa (Barth et al., 2010). Drug resistance viruses is a major factor contributing to treatment failure and develops when viral replication continues in the presence of selective drug pressure and the cause should be the impotent regimens, ineffectively treated compartments, suboptimal adherence and pharmacological hurdles(Bulage et al., 2017).In Burundi, many interventions for control and prevention, such as PMTCT, care services for treatment with ARV drugs have been implemented. As of 2014, the most recent year for which an estimate is available, $1.3 \%$ of the population in Burundi was living with HIV with approximately 4,700 annual deaths attributed to AIDS. According to the Spectrum estimates (Version 2015), the number of people living with HIV (PLHIV) in Burundi was 81,965 (adults and children), amounting to an estimated ART coverage of $51 \%$ (56\% among adult, and $17 \%$ among children) (Summary, 2016).To date, the use of CD4 count and Viral load testing in Burundi have been implemented as monitoring of treatment failure but little is known about the prevalence of virological failure and patterns of drug resistance in HIV-infected patients in Burundi. Our study was aimed at determining the prevalence of virological failure and describing drug resistance mutations in HIV-1 infected patients on ART over 12 months of first line therapy, attending Kayanza district hospital, Burundi. 


\section{Material and Methods}

2.1. Study design

This was a descriptive cross sectional study.

\subsection{Study site}

This study was carried out at Kayanza District Hospital; located at Kayanza Centre, in Kayanza Province, Burundi. It is the capital city of Kayanza Province.

\subsection{Study population}

These were HIV-1 infected patients on ART over 12 months attending Kayanza district hospital. The study population included patients living with HIV and AIDS, males and females, from 2 year old and over, who were on first line ART for more than 12 Months.

\subsection{Epidemiological data collection}

A questionnaire was used to collect demographic data, clinical, drugs experienced and any other characteristics. More patients' information's were also obtained through desk review of the clinical and laboratory records from the patient's files.

\subsection{Sample collection and handling}

This was a purposive sampling method where all patients who met the inclusion criteria were recruited into the study. They were requested to voluntary sign the informed consent before enrolled in the study. Up to $4 \mathrm{~mL}$ of venous blood was collected from each patient in Ethylene diamine tetra acetic Acid (EDTA) tubes and shipped to Ngonzi regional hospital and Burundi National Reference Laboratory (NRL) for further processing. Plasma with suspected virological failure (Viral load $>1000$ copies $/ \mathrm{mL}$ ) were stored at $-80^{\circ} \mathrm{C}$ and later shipped to the Kenya Medical Research Institute (KEMRI), in Nairobi - Kenya, where further testing and analysis were performed.

\subsection{Statistical analysis}

Analysis were done using SPSS version 21. We used descriptive statistics to analyse socio-demographic data, clinical and immunological outcomes and virological outcomes. The data was reported as percentages. Multinomial logistic regression was used to adjust for odds of viral outcomes associated with various demographic factors (like gender, age, religion, education status, marital status, income source, presence of other partners and reminders to take ARVs) as well as other medical factors (like taking alcohol, irregularity in taking drugs, taking TB drugs, line of ARV medication, date of staring treatment, CD4 initial count and CD4 count at study time). The results were expressed as odds ratios. (OR) with $95 \%$ confidence intervals (CIs).

\subsection{Ethical clearance}

The study was approved by the Burundi National Ethical Committee (BERC: 25/03/2018). To maintain the confidentiality, a unique ID number was given to each specimen, and written consent or assent from each patient was obtained. No names or direct identifiers of the participants were published anywhere.

\subsection{Viral load testing}

Viral load assay for plasma samples was done using Real Time HIV-1 assay Kit Abbott molecular Inc.; Des Plaines, IL 60018, USA), which has an internal Quality Control system, with automated Abbott $m 2000 r t^{\mathrm{TM}}$ System and was perfomed according to the manufacturer's instruction. Lower limit of detection was 40 copies of HIV RNA / mL.Samples with viral load > 1000 copies/ml were considered for Sequencing and Genotyping.

\subsection{Extraction of RNA and Reverse Transcription}

The RNA was extracted from plasma samples using the Qiagen RNA extraction Kit (QIAamp® Viral RNA mini kit Qiagen RNA extraction protocol) according to the manufacturers' instructions (Luvai et al., 2015).The template $(10 \mu \mathrm{l}$ of extracted RNA) was reverse transcribed in a total volume of $20 \mu \mathrm{l}$ with $1 \mu \mathrm{lNTP}, 2 \mu \mathrm{l}$ primer, $4 \mu \mathrm{l} 5 \times$ RT buffer, $2 \mu \mathrm{l}$ DTT, $0.25 \mu$ l Ranse OUT and $1.0 \mu 1$ SuperScriptTM III RNase H- RT (Invitrogen, Carlsbad, CA2). The RNA, primer and dNTPs were first incubated at $65^{\circ} \mathrm{C}$ for 5 minutes, then $4^{\circ}$ for 5 minutes, and the remaining reagents were added for cDNA synthesis at $25^{\circ} \mathrm{C}$ for 5 minutes, $50^{\circ} \mathrm{C}$ for 40 minutes, followed by $70^{\circ} \mathrm{C} 15$ minutes (Khamadi et al., 2005).

\subsection{Polymerase Chain Reaction (PCR)}

The cDNA was used as template to amplify the region of interest for HIV-1 drug resistance. The reagents were thawed on ice to preserve their integrity. The total volume of outer PCR was $25 \mu \mathrm{L}$ reaction volume with a 
mixture containing $3 \mu \mathrm{L}$ of the DNA template to be amplified, $2.5 \mu \mathrm{L}$ of $10 \mathrm{X}$ buffer (without magnesium chloride) ,0.3 $\mu \mathrm{L}$ of each RT18 (RT 18(5'-GGAAACCAAAAATGATAGGGGGAATTGGAGG-3') ) and KS104 primers (KS 104(5'-TGATTGCCCAATTTAGTTTTCCCACTAA-3'), 0.5 $\mu$ L dNTP, $2.0 \mu \mathrm{L}$ Mgcl2, 0.2 $\mu \mathrm{L}$ of Taq polymerase and $16.2 \mu \mathrm{L}$ of distilled water(Nyamache et al., 2011). The cycling conditions were 1 cycle of $95^{\circ} \mathrm{C}$ for $10 \mathrm{~min}$ and 35 cycles of $95^{\circ} \mathrm{C}$ for $30 \mathrm{~s}$, annealing at $55^{\circ} \mathrm{C}$ for $30 \mathrm{~s}$, and $72^{\circ} \mathrm{C}$ for $1 \mathrm{~min}$, and final extension of $72^{\circ} \mathrm{C}$ for $10 \mathrm{~min}$. From the first-round PCR products, $3 \mu \mathrm{L}$ was used as a template to make $25 \mu \mathrm{L}$ for the second reaction volume with the second set of primers ;KS101(KS101(5'GTAGGACCTACACCTGTTCAACATAATTGGAAG-3') KS102 and KS102(5' CCCATCCAAAGAAATGGAGGAGGTTCTTTCTGATC-3') using the same cycling conditions(Nyamache et al., 2011). The nested PCR product were run on a 2\% agarose gel and electrophoresed at a voltage of 100 for 20 minutes(Kiptoo et al., 2013).

\subsection{Sequencing}

The amplicons from the nested PCR were sequenced. Using the ABI 3730xl DNA Analyzer (96 capillary type), Sequencing reactions were performed in the DNA Master cycler pro 384(Eppendorf) using the ABI BigDye ${ }^{\circledR}$ Terminator v3ext Cycle Sequencing Kit (Applied Biosystems). Single-pass sequencing was performed on each template using selected primers. The fluorescent-labeled fragments were purified from the unincorporated terminators with the BigDye XTerminator ${ }^{\circledR}$ Purification Kit (Applied Biosystems). The samples were injected to electrophoresis in an ABI 3730xl DNA Analyzer (Applied Biosystems).A reaction mixture containing the following reagents was used for sequencing PCR: Thirteen (13) $\mu$ l RNase free double distilled $\mathrm{H}_{2} \mathrm{O}, 5 \mathrm{x}$ sequencing buffer, $1.5 \mu \mathrm{l}$ primers of $1 \mu \mathrm{M}$ sense and antisense primer, $1.0 \mu \mathrm{l}$ big dye terminator and $1.0 \mu \mathrm{PCR}$ product making up a total reaction volume of $20 \mu \mathrm{l}$ (Songok et al., 2004). The PCR tubes containing the samples were placed into the thermocycler under the following programmed conditions; Initial PCR activation step $96^{\circ} \mathrm{C}$ for 2 minutes; 30 cycles of: denaturation for 10 seconds at $96^{\circ} \mathrm{C}$, annealing for 10 seconds at $50^{\circ} \mathrm{C}$; extension for 4 minute at $60^{\circ} \mathrm{C}$ and thereafter the samples were held at $4^{\circ} \mathrm{C}$ (Kiptoo et al ., 2013; Lihana et al., 2009) .

\subsection{HIV-1 Subtyping}

The Sequences derived in this study were subjected to BLAST and REGA search tools to determine subtype diversity. Reference sequences were selected from Los Alamos HIV database. The sequences were aligned and gaps removed using BioEdit. The tree topology was obtained using the neighbour-joining method .A phylogenetic tree was constructed using neighbour joining method in MEGA 6 software (Tamura et al., 2013). Alignment of DNA sequences was performed using the CLUSTALW program (http://www.clustal.org). The pairwise distance matrix was estimated using the Kimura two parameter model with the MEGAv6 software package (http://www.megasoftware.net). Bootstrap_re-sampling (1000 data sets) of the multiple alignments was performed to set the statistical robustness of the tree. The tree profile was visualized with tree view PPC version 1.65 (Foley et al., 2015.)

\subsection{Genotypic drug resistance mutations identification}

Drug Resistance mutations, defined using the 2018 update of the International AIDS Society (IAS) USA, and were manually located in each RT sequence following the IAS-USA nomenclature. Genotypic interpretation of the resistance mutations was carried out using the Stanford HIVdb Algorithm, version 6.0.11 (Stanford University, Palo Alto, CA).Drug resistance was categorized in the tree levels: Susceptible(S),Intermediate(I) and Resistant (R).All sequences associated with high level resistance according to Stanford IAS Algorithms were considered to be resistance.

\section{Results}

\subsection{Baseline characteristics of the study participants}

A total of 200 patients who met the inclusion criteria were included in the study. All patients were ARV experienced based on 2008 Burundi National guidelines, which proposed combination of Zidovudine (AZT), lamivudine (3TC) and Nevirapine or Efavirenz are the most commonly prescribed first-line. The age distribution was unimodal and it peaked at 45 years (range 3 to 67 years). More than half of the respondents ( $\mathrm{n}=172$ ) were above 18 years and female accounted for $55 \%(n=110)$. Stratification by religion, $73.5 \%(n=147)$ were christian catholics while $52 \%(n=104)$ were married On the level of education, $82.5 \%(n=165)$ reported completing primary school while farming as occupation was reported by $65 \%(n=131)$ with $94 \%(n=188)$ reporting to earn below BIF 200.The study realized that $12.5 \%$ (25) of the patients were on tuberculosis (TB) medications while $57 \%$ (114) had been on antiretroviral (ARV) therapy for between six to ten years. In total 51\%, (102) did not have detectable viral loads while $\left(39 \%,(78)\right.$ had a CD4 count of $>350$ cells $/ \mathrm{mm}^{3}$. While at the time of starting the ARV medications $68 \%$ (137) of the respondents had a CD4 count of $>350$ cells $/ \mathrm{mm}^{3}$. text On ARV medication, 
$56.5 \%$ (113) of the respondents reported using tenofovir (TDF), lamividune (3TC) and Efavirenz (EFV) drug regimen, but TDF,tenofovor-3TC,lamividune-NVP,nevirapine;zidovudine $3 \mathrm{TC}+\mathrm{NVP}$,nevirapine; Duovir; Duovir+ EFV, efavirenz were also used .

AZT+lamividune,

\subsection{Virological outcomes}

At the time of the study, 51\% (102/200) of the respondents did not have detectable viral load (VL $<40$ copies/ml), 34\%, n=72/200 were between 400 and 999 copies/ml while 13\%, (26/200) had virological failure (VL $\geq 1000$ copies $/ \mathrm{ml}$ ). Virological failure was strongly with the presence of other partners; $p=0.016$ (Table 1).

Table 1. Relationship between virological failure and demographic characteristics among HIV positive patients attending Kayanza district Hospital $(n=26)$.

\begin{tabular}{|c|c|c|c|c|}
\hline \multicolumn{2}{|l|}{ Variable } & Viral Failure & aOR & P Value \\
\hline \multirow{2}{*}{ Gender } & Male & 14 & \multirow{2}{*}{1.2} & \multirow{2}{*}{0.337} \\
\hline & Female & 12 & & \\
\hline & & 25 & & \\
\hline \multirow{3}{*}{ Age } & 12-Feb & 4 & 0.8 & 0.915 \\
\hline & $13-17$ & 3 & 0.994 & 0.997 \\
\hline & $>18$ years & 19 & 1 & \\
\hline \multirow{3}{*}{ Religion } & Catholic & 18 & 0.7 & 0.566 \\
\hline & Protestant & 1 & 0.3 & 0.182 \\
\hline & Muslim & 7 & 1 & \\
\hline \multirow{3}{*}{ Educational status } & primary & 22 & 0.62 & 0.256 \\
\hline & secondary & 3 & 0.87 & 0.732 \\
\hline & university & 1 & 1 & \\
\hline \multirow{4}{*}{ Marital Status } & Single & 9 & 3 & 0.504 \\
\hline & Married & 7 & 0.13 & 0.667 \\
\hline & Divorced & 2 & 0.2 & 0.142 \\
\hline & Widowed & 8 & 1 & \\
\hline \multirow{3}{*}{ Income source } & Farmer & 15 & 1.1 & 0.943 \\
\hline & Formal employment & 1 & 1.7 & 0.673 \\
\hline & Informal employment & 10 & 1 & \\
\hline \multirow{2}{*}{ Missing dose } & Yes & 3 & 0.154 & 0.016 \\
\hline & No & 23 & 1 & \\
\hline \multirow{2}{*}{ Reminded to take ARVs } & Yes & 22 & 4.3 & 0.072 \\
\hline & No & 4 & 1 & \\
\hline
\end{tabular}

Viral failure was significantly correlated with irregularity in taking medications (adjusted odds ratio (aOR):0.4, $\mathrm{p}=\mathbf{0 . 0 1 4})$ (Table 2).

Table 2.Relationship between viral failure and other medical and social factors among HIV positive patients attending Kayanza district Hospital $(n=26)$.

\begin{tabular}{|l|l|l|l|l|}
\hline Variable & Ves & Viral Failure & aOR & P Value \\
\hline \multirow{2}{*}{ Taking alcohol } & No & 6 & 0.8 & \multirow{2}{*}{0.711} \\
\hline \multirow{2}{*}{ Missing Dose } & Yes & 20 & 1 & \multirow{2}{*}{$\mathbf{0 . 0 1 4}$} \\
\cline { 2 - 5 } & No & 5 & 0.4 & \multirow{2}{*}{0.251} \\
\hline \multirow{2}{*}{ Taking TB Drugs } & Yes & 21 & 1 & \multirow{2}{*}{0.075} \\
\cline { 2 - 5 } & No & 4 & 0.3 & 0.81 \\
\hline \multirow{3}{*}{ Time of ARV Medication } & First line*** & 23 & 1 & 0.994 \\
\cline { 2 - 5 } & Second line** & 17 & 5.2 & \\
\hline \multirow{3}{*}{ CD4 initial count } & 1 & 9 & 1 & 0.412 \\
\cline { 2 - 5 } & 2 & 10 & 0.7 & 0.833 \\
\cline { 2 - 5 } & 3 & 1 & 1.1 & \\
\hline \multirow{3}{*}{ CD4 Count at study time } & $<200$ cells $/ \mathrm{mm}^{3}$ & 9 & 1.8 & 0.598 \\
\cline { 2 - 5 } & $200-350 \mathrm{cells} / \mathrm{mm}^{3}$ & 7 & 0.881 & 1 \\
\cline { 2 - 5 } & $>350$ cells $/ \mathrm{mm}^{3}$ & 10 & 1 & \\
\cline { 2 - 5 } & $200-350 \mathrm{cells} / \mathrm{mm}^{3}$ & 6 & 0.6 & \\
\cline { 2 - 5 } & $>350$ cells $/ \mathrm{mm}^{3}$ & 17 & & \\
\hline
\end{tabular}




ISE

\begin{tabular}{|lc|}
\hline$* * *$ First line drug regimen & $* *$ Second line drug regimen \\
\hline Adolescent and adults & \\
\hline 2NRTI+NNRTI & 2NRTI+PI/r \\
\hline TDF/3TC/EFV (first choice) t & $\mathrm{ABC} / 3 \mathrm{TC}+\mathrm{ATV} / \mathrm{r}$ (first choice) \\
\hline TDF/3TC+ NVP (alternative) & $\mathrm{ABC} / 3 \mathrm{TC}+\mathrm{ATZ} / \mathrm{r}$ (alternative) \\
\hline AZT/3TC/NVP (alternative) & $\mathrm{ABC} / 3 \mathrm{TC}+\mathrm{ATZ} / \mathrm{r}$ (alternative) \\
\hline Under 3 years: & \\
\hline NRTI & NNRTI \\
\hline ABC+3TC+LPV/r (first choice) & $\mathrm{AZT}+3 \mathrm{TC}+\mathrm{RAL}$ (first choice) \\
\hline ABC+3TC+LPV/r over 3 (second line) & $\mathrm{ABC}+3 \mathrm{TC}+\mathrm{RAL}$ (alternative) \\
\hline 3-10 years: & \\
\hline NRTI & NNRTI \\
\hline ABC+3TC+EFV (first choice) & AZT $+3 T C+A T V / r$ (first choice) \\
\hline $\begin{array}{l}\text { ABC+3TC+NVP (alternative) } \\
\text { ABC+3TC+ATV/r (alternative) }\end{array}$ & AZT+3TC+ATV/r (alternative) AZT+3TC+NVP (alternative \\
\hline
\end{tabular}

\subsection{Phylogenetic characterization of HIV-1 variants}

Genotype sequencing was performed on 26 samples with virological failure (HIV-1 RNA $\geq 1,000 \mathrm{cop} / \mathrm{mL}$ ). Fifteen (15) samples were successfully sequenced. From these, $80 \%(12 / 15)$ were HIV-1 subtype C, 7\% (1/15) were subtype A, and 13\% (2/15) were HIV-1 subtype A1 (Table 2). The Phylogenetic tree, illustrated with reference sequences labelled using their gene bank accession numbers and country of origin show that HIV-1 subtype C clustered with those of Burundi, Rwanda, and Tanzania. The samples with subtype A clustered with those from Burundi and DRC (Figure.1).

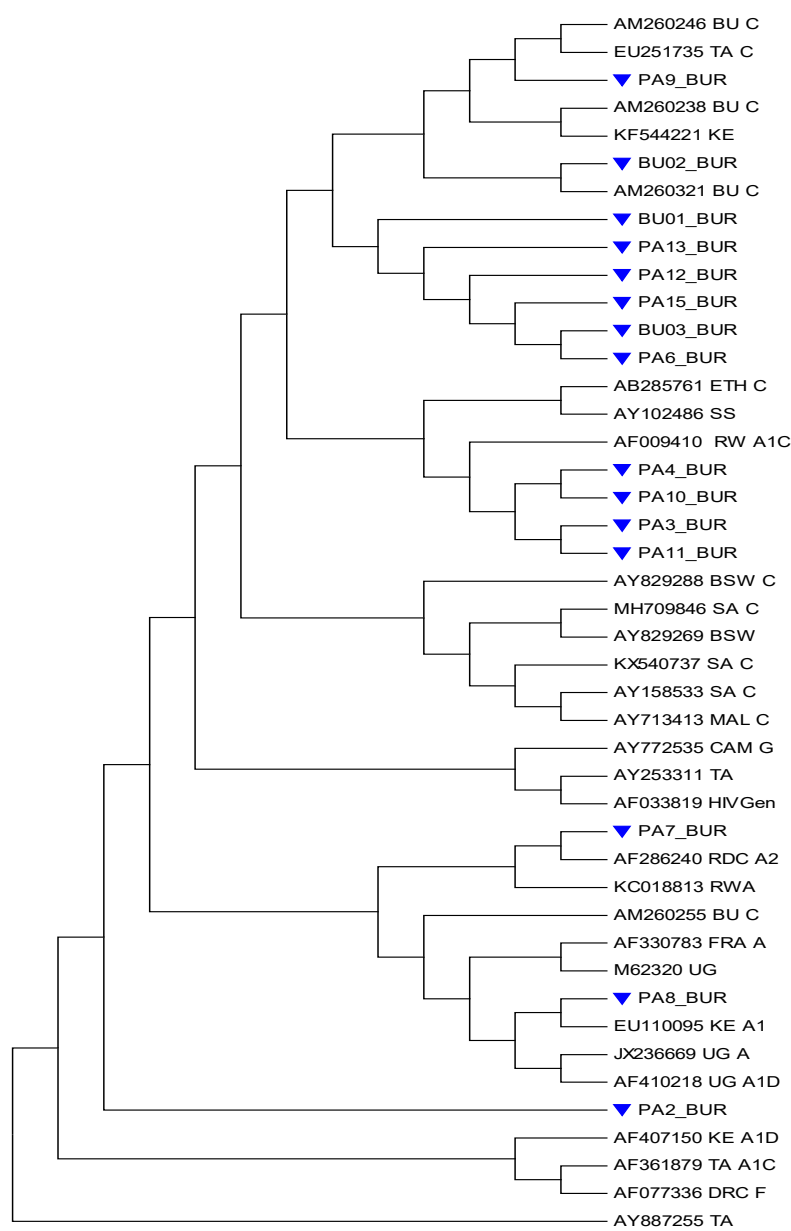

Figure1. Phylogenetic tree illustrated using the samples with reference sequences from Los Alamos data base. 
The above phylogenetic tree illustrating the evolutionary relationships of HIV-1 subtypes found from the samples in relation to reference sequence from Los Alamos database. The samples are labeled with PA_BUR and PA_BUR with blue triangle followed by the number and subtype and the references are labeled by their accessory number, the subtypes and the country of origin.

\subsection{Drug resistance mutations analysis}

Our study showed that $80 \%(12 / 15)$ had at least one mutation (NRTI or NNRTI), while 20\% (3/15) did not carry any drug resistance mutations. Based on drug class, the most common drug resistance mutations were against NNRTI $80 \%$ (12/15) while DRM against NRTI were $47 \% 7 / 15$. The most common drug resistance mutations were M183V, M41L, E44D, L74I, L210W, K65R, K103N, E188A and Y188H (Table 3). Finally, minor mutations were found like V179I in two patients, K101Q in 5 patients and S68G in one patient. For NRTIs, the HIV drug resistance mutations found were against FTC, 3TC, D4T and TDF while for the NNRTI the mutations found were against EFV and NVP (Table 3).

Table 3. Resistance mutations by drug class and subtypes in HIV patients attending Kayanza district hospital.

\begin{tabular}{|c|c|c|c|c|c|}
\hline \multirow[t]{3}{*}{$\begin{array}{c}\text { ID } \\
\text { Sequences } \\
\end{array}$} & $\begin{array}{c}\text { Subtype } \\
\text { s } \\
\end{array}$ & \multicolumn{4}{|c|}{ DRMs } \\
\hline & & \multicolumn{2}{|c|}{ NRTIs } & \multicolumn{2}{|l|}{ NNRTIs } \\
\hline & & HDRMs & DRUGS & HDRMs & DRUGS \\
\hline BU01_BUR & $\mathrm{C}$ & M184V & FTC,3TC & $\mathrm{K} 103 \mathrm{~N}$ & NVP,EFV \\
\hline BU02_BUR & $\mathrm{C}$ & None & - & $\mathrm{K} 103 \mathrm{~N}$ & NVP,EFV \\
\hline BU03_BUR & $\mathrm{C}$ & M184V & FTC,3TC & K103N, Y188H & EFV,NVP \\
\hline PA2 BUR & A & K65R,M184V & $\begin{array}{c}\text { FTC,ABC,DD } \\
\text { I }\end{array}$ & K103N, & EFV,NVP \\
\hline PA3_BUR & $\mathrm{C}$ & None & - & K103N & EFV,NVP \\
\hline PA4_BUR & $\mathrm{C}$ & None & - & $\mathrm{K} 103 \mathrm{~N}$ & EFV,NVP \\
\hline PA6_BUR & $\mathrm{C}$ & M184V & 3TC,FTC & $\mathrm{K} 103 \mathrm{~N}$ & EFV,NVP \\
\hline PA7_BUR & A1 & None & - & None & None \\
\hline PA8_BUR & A1 & None & - & None & - \\
\hline PA9_BUR & $\mathrm{C}$ & None & - & None & - \\
\hline PA10_BUR & $\mathrm{C}$ & None & - & $\mathrm{K} 103 \mathrm{~N}$ & EFV,NVP \\
\hline PA11_BUR & $\mathrm{C}$ & None & - & $\mathrm{K} 103 \mathrm{~N}$ & EFV,NVP \\
\hline PA12_BUR & $\mathrm{C}$ & M184V & FTC,3TC & $\mathrm{K} 103 \mathrm{~N}$ & EFV,NVP \\
\hline PA13_BUR & $\mathrm{C}$ & $\begin{array}{l}\text { M41L,E44D,L74I, } \\
\text { M184V,L210W }\end{array}$ & $\begin{array}{c}\text { ABC,FTC,DD } \\
\text {,3TC }\end{array}$ & K103N,E138A & EFV,NVP \\
\hline PA15_BUR & $\mathrm{C}$ & M184V & FTC,3TC & $\mathrm{K} 103 \mathrm{~N}$ & EFV,NVP \\
\hline
\end{tabular}

\section{Discussion}

To our knowledge this was the first descriptive study on virological and HIV drug resistance mutations outcomes conducted in HIV patients after 12 months of treatment in Burundi. All patients were ARV experienced based on 2008 Burundi National guidelines, which proposed combination of Zidovudine (AZT), lamivudine (3TC) and Nevirapine or Efavirenz as the commonly prescribed first-line treatment combinations. In this unimodal population with $86 \%$ above 18 years with $55 \%$ mainly females, the treatment failure of $13 \%$ raises a concern to the public health system, as low level transmitted resistance could already be taking place in the general population. This success of HIV drug treatment a met the WHO recommendation of $>85 \%$ of patients on ART suppressing HIV-1 VL after 12 months of treatment. At $87 \%$ viral suppression after 12 months on treatment, Burundi exhibits a potential to reach the third $90 \%$ of the $90-90-90$ target recently set by UNAIDS Likewise, studies conducted in other countries in Resources Limited Settings such as Rwanda, Tanzania show virological suppressed of $88.1 \%$ and $86 \%$ respectively (Ndahimana et al., 2016; Rusine et al., 2013; Hawkins et al., 2016) but are high contrary to those found in Latine America and in Ethiopia. (Cesar et al., 2015; Endalamaw et al., 2018). The reason for geographic area where carried the study should not be excluded in this discordant findings .In our study, Viral failure was significantly correlated with irregularity in taking medications missing dose. As Kayanza Hospital is a rural health facility, poor adherence to distance-related antiretroviral therapy may be observed and could certainly lead to virological failure. The findings highlight why adherence is good, not only for the prevention of morbidity, mortality and transmission, but also for the prevention of HIV drug resistance. Our results are not surprising because it has long been known that low adhesion leads to virological failure and resistance to anti-HIV drugs. (Luvai et al. ,2015; Etta et al., 2017, Endalamaw et al., 2018).Although, even the national HIV program in Burundi provides basic membership counseling, outreach activities should be 
planned to improve adherence interventions for ARV patients and minimize follow-up losses. Our finding show also association between virological failures with the presence of other partners. This should be the evidence from HIV patients on ARV who have sex with those who have already the acquired or transmitted drug resistance. Also, multiple infections with different strains of the virus and other sexually transmitted infections can lead to ART drug resistance hence virological failure (Dessie.,2011). This could potentially be due to transmission of resistance virus from other partners, an assertion that calls for further investigation of the rate of transmitted resistance. In our study, fifteen samples were successfully sequenced; $80 \%(12 / 15)$ were HIV-1 subtype C, 7\% (1/15) were subtype A, and 13\% (2/15) were HIV-1 subtype A1. HIV-1 subtype C is the most prevalent worldwide and is the major HIV-1 subtype non subtype B in some countries of southern and eastern Africa, Asia and the Middle East, where most infections occur ( Luvai et al.,2015). Our findings concurred with previous studies conducted in Burundi (Vidal et al., 2007;) and have been reported with an important fraction of HIV infection in east Africa(Delatorre \& Bello., 2012; Lihana et al.,2012).Also sexual transmission of this subtype is also suggested to be more than that of subtype A (Abecasis et al., 2013). Althoug, our finding is particularly not similar to those found in Rwanda, one of borders country (Rusine et al., 2013). This should mention the absence of a cross transmission from these two countries. We suggest that a study should be done to reveal the origin of that predominant of subtype $\mathrm{C}$ in Burundi, wich is not predominant in borders countries. (Rusine et al., 2013; Luvai et al., 2015)From this study, subtype C was observed to have a high number of drug associated mutations. The M184V was the highest mutation (33\%) in NRTI and is known to confer very high resistance against NRTI. For NRTI, Subtype C viruses develop resistance against non-nucleoside reversetranscriptase inhibitors of $\mathrm{K} 103 \mathrm{~N}$ at $77 \%$ and this are known to be common in cases of treatment failure ( Rusine et al., 2013). Also, Grossman et al., 2004 in their study showed that Subtype C viruses develop resistance against nonnucleoside reverse-transcriptase inhibitors through the K103N mutations. Due to the small number of sample size, it is difficult to determine effective treatment of the different subtypes. Our finding showed that $80 \%(12 / 15)$ had at least one NRTI or NNRTI. One reason might have been the low sub-optimal adherence. Our results indicate that reliable measurements of drug adherence are needed. This results should not be unexpected as the drug resistance mutations was done from patients with virological failure .Also, the drug resistance mutation between $79 \%$ to $85 \%$ have been reported from many authors in sub-Saharan Africa like in Rwanda, Kenya and Tanzania ( Rusine et al., 2013., Luvai.,2016.; Hawkins et al., 2016) . Althoug $20 \%$ (3/15) of our findings did not carry any drug resistance mutations. Based on drug class, the most common DRM were against NNRTI $(81.25 \%)$ while DRM against NRTI were $62.5 \%$.Also, the most common mutations in this study were $\mathrm{M} 184 \mathrm{~V}$, K65R in NRTI; K103N and Y188H in NNRTI. Our finding is common as long as these mutations are known to be common in cases of treatment failure and have been reported elsewhere (Johnson et al., 2013). They are associated with the use of 3TC, EFV and NVP, which have low genetic barriers towards resistance. The M184V wich his prevalent is known to cause resistance to 3TC and FTC, enhances the susceptibility to AZT, and delays the emergence of mutations associated with AZT and d4T (Johnson et al., 2013).His relatively high overall prevalence in this study may have consequences for second line treatment responses in HIV patients attending Kayanza district hospital. We also mention in our study the presence of K103N and Y188H which are more likely present in subtypes C (Delatorre \& Bello, 2012) ..For NRTIs, the HIV drug resistance mutations found were against FTC, 3TC, and TDF while for the NNRTI the mutations found were against EFV and NVP) .This should be explained by the use of regimen based in 2NRTI+ 1NNRTI in the first line therapy. The utility of these agents as part of second- or third-line regimens is limited and then this should have an impact on second-line nucleoside analog therapy options with consequences of limit future treatment options. Also, we found HIV drug resistance mutations against D4T which have not even been introduced in Burundi Guide line. Further resistance testing on this drug regimen should be conducted before its introduction as a first line regimen in Burundi. In 15samples successfully sequenced, only 2 were patients under 18 years, from which did not harbour any mutation both in NRTIs and NNRTIs. The children should develop the transmitted or acquired drug resistance mutations. Although, due to the relatively small number of children enrolled in our study, these findings should not rule out presence of transmitted or acquired drug resistance mutations in HIV positive children at kayanza district hospital. A specific study with high number of children should be conducted to confirm our findings. Although our findings may not be generalized to all HIV clinics in Burundi but should indicate that some strategies to minimize HIVDR are needed .These should include improved availability and utilization of VL-based monitoring of ART response, and evaluation of the potential added value of HIV genotyping at treatment failure .In addition, high quality patient support for treatment adherence will contribute to protecting the efficacy of second line and subsequent therapy and improving overall treatment outcome .

\section{Conclusion}

In the conclusion, our study findings highlight the critical importance of Viral Load monitoring in Burundi programmes health-care and can help detecting Virological Failure earlier before extensive development of multidrug resistance which can render second- line unsuccessful and ineffective. Also, our study population had 
high number of resistance mutations to all the NRTIs and NNRTIs used in HIV treatment in Burundi health care and the consequence is extremely high as long as none of the proposed drugs for third -line use are available. Effective monitoring of HIV positive patients using viral loads and drug resistance mutations surveillance is critical.Our study was a case control with a relatively small sample size of only 200 and thus may not be fully representative to the entire patients on ART in Burundi. Additional more robust studies as longitudinal cohort studies with high number of patients are needed to confirm our finding on virological outcomes and drug resistance mutations in HIV patients in Burundi.

\section{Conflict of interest declaration.}

The authors declare that they have no competing interests

\section{Genbank Accession Number: MK580981-MK580995.}

\section{Acknowledgment}

This study was funded by the East Africa Public health Laboratory Network Project (EAPHLNP)/Burundi. The study also received technical support from the Kenya Medical Research institute. We are grateful also to all staff of Ngozi hospital, Kayanza hospital and Burundi National Reference Laboratory for their help in sample collection, processing and storage.

\section{REFERENCES}

Barth, R. E., van der Loeff, M. F. S., Schuurman, R., Hoepelman, A. I., \& Wensing, A. M. (2010). Virological follow-up of adult patients in antiretroviral treatment programmes in sub-Saharan Africa: a systematic review. The Lancet Infectious Diseases, 10(3), 155-166.

Bello, G., Passaes, C. P., Guimarães, M. L., Lorete, R. S., Matos Almeida, S. E., Medeiros, R. M., Morgado, M. G. (2008). Origin and evolutionary history of HIV-1 subtype $\mathrm{C}$ in Brazil: AIDS, 22(15), 1993-2000.

Bulage, L., Ssewanyana, I., Nankabirwa, V., Nsubuga, F., Kihembo, C., Pande, G., Kiyaga, C. (2017). Factors associated with virological non-suppression among HIV-positive patients on antiretroviral therapy in Uganda, August 2014-July 2015. BMC Infectious Diseases, 17(1), 326.

Cesar, C., Jenkins, C. A., Shepherd, B. E., Padgett, D., Mejía, F., Ribeiro, S. R., Cahn, P. (2015). Incidence of virological failure and major regimen change of initial combination antiretroviral therapy in the Latin America and the Caribbean: an observational cohort study. The Lancet HIV, 2(11), e492-e500.

Delatorre, E. O., \& Bello, G. (2012). Phylodynamics of HIV-1 Subtype C Epidemic in East Africa. PLoS ONE, 7(7), e41904.

Dessie, Y., Gerbaba, M., Bedru, A., \& Davey, G. (2011). Risky sexual practices and related factors among ART attendees in Addis Ababa Public Hospitals, Ethiopia: A cross-sectional study. BMC Public Health, $11(1)$.

Endalamaw, A., Mekonnen, M., Geremew, D., Ambaw, F., Tesera, H., \& Habtewold, T. D. (2018). Evidence that poor HAART adherence has a great impact on HIV/AIDS treatment failure more than severity of illness and opportunity of infection in Ethiopia: Systematic review and meta-analysis. BioRxiv. https://doi.org/10.1101/440743

Etta, E. M., Mavhandu, L., Manhaeve, C., McGonigle, K., Jackson, P., Rekosh, D., T ebit, D. M. (2017). High level of HIV-1 drug resistance mutations in patients with unsuppressed viral loads in rural northern South Africa. AIDS Research and Therapy, 14(1).

Foley, B., Leitner, T., Apetrei, C., Hahn, B., Mizrachi, I., Mullins, J. Yoon, H. (2015). HIV Sequence Compendium 2018, 450.

Grossman, Z., Istomin, V., Averbuch, D., Lorber, M., Risenberg, K., Levi, I., \& Israel AIDS Multi-Center Study Group. (2004). Genetic variation at NNRTI resistanceassociated positions in patients infected with HIV-1 subtype C. Aids, 18(6), 909- 915.

Hamers, R. L., Sigaloff, K. C., Kityo, C., Mugyenyi, P., \& de Wit, T. F. R. (2013). Emerging HIV-1 drug resistance after roll-out of antiretroviral therapy in sub-Saharan Africa. Current Opinion in HIV and AIDS, $8(1), 19-26$.

Hawkins, C., Ulenga, N., Liu, E., Aboud, S., Mugusi, F., Chalamilla, G. Fawzi, W. (2016). HIV virological failure and drug resistance in a cohort of Tanzanian HIV-infected adults. Journal of Antimicrobial Chemotherapy, 71(7), 1966-1974.

Johnson, V. A., Calvez, V., Günthard, H. F., Paredes, R., Pillay, D., Shafer, R. W. Richman, D. D. (2013). Update of the Drug Resistance Mutations in HIV-1: March 2013, 2.

Kiptoo, M., Brooks, J., Lihana, R. W., Sandstrom, P., Ng'ang'a, Z., Kinyua, J., Songok, E. M. (2013). HIV1 drug resistance-associated mutations among HIV-1 infected drug-naïve antenatal clinic attendees in rural Kenya. BMC Infectious Diseases, 13(1). https://doi.org/10.1186/1471-2334-13-517 
LeL Rency (2018). Molecular characterization of HIV and drug resistance mutations among HIV positive children attending Busia county referral hospital, Kenya, 81.

Lihana, R. W., Khamadi, S. A., Lubano, K., Lwembe, R., Kiptoo, M. K., Lagat, N., \& Ichimura, H. (2009). HIV type 1 subtype diversity and drug resistance among HIV type 1-infected Kenyan patients initiating antiretroviral therapy. AIDS research and human retroviruses, 25(12), 1211-1217.

Lihana Raphael W, Deogratius Ssemwenga, Alash'le Abimiku, and Nicaise Ndembi. (2012). HIV-1 Diversity in Africa. A decade in review. AIDS, 14:83-100.

Luvai, E. A. C. (2013.). Evaluation of predictors of HIV/AIDS treatment failure in patients with suspected virological and immunological failure from comprehensive care clinic in Nairobi, 2013, 89.

Ndahimana, J. d'Amour, Riedel, D. J., Mwumvaneza, M., Sebuhoro, D., Uwimbabazi, J. C., Kubwimana, M., Nsanzimana, S. (2016). Drug resistance mutations after the first 12 months on antiretroviral therapy and determinants of virological failure in Rwanda. Tropical Medicine \& International Health, 21(7), 928935.

Nsanzimana, S., Remera, E., Kanters, S., Chan, K., Forrest, J. I., Ford, N. Mills, E. J. (2015). Life expectancy among HIV-positive patients in Rwanda: a retrospective observational cohort study. The Lancet Global Health, 3(3).

Nyamache AK, Waihenya R., Muigai AW and Khamadi SA. (2011). Reverse Transcriptase Inhibitors Drug Resistance Mutations in Drug-Naive HIV Type 1 Positive Kenyan Individuals. East African medical journal, 88(1)4-8.

Rusine, J., Asiimwe-Kateera, B., van de Wijgert, J., Boer, K. R., Mukantwali, E., Karita, E., Ondoa, P. (2013). Low Primary and Secondary HIV Drug-Resistance after 12 Months of Antiretroviral Therapy in Human Immune-Deficiency Virus Type 1 (HIV-1)-Infected Individuals from Kigali, Rwanda. PLoS ONE, 8(8), e64345. https://doi.org/10.1371/journal.pone.0064345

Summary, S. D. (2016). PEPFAR Burundi, 1-46.

Tamura, K., Stecher, G., Peterson, D., Filipski, A., \& Kumar, S. (2013). MEGA6: Molecular Evolutionary Genetics Analysis Version 6.0. Molecular Biology and Evolution, 30(12), 2725-2729.

Vidal, N., Niyongabo, T., Nduwimana, J., Butel, C., Ndayiragije, A., Wakana, J., Peeters, M. (2007). HIV Type 1 Diversity and Antiretroviral Drug Resistance Mutations in Burundi. AIDS Research and Human Retroviruses, 23(1), 175-180.

Ssemwanga, D., von Braun, A., Sekaggya-Wiltshire, C., Bachmann, N., Scherrer, A. U., Nanyonjo, M., Kambugu, A. (2018). HIV-1 Drug Resistance among Ugandan Adults Attending an Urban Out-Patient Clinic: JAIDS Journal of Acquired Immune Deficiency Syndromes, 78(5), 566-573. 